\title{
Secretory expression of Par-4 SAC-HA2TAT following adeno-associated virus-mediated gene transfer induces apoptosis in HepG2 cells
}

\author{
TIAN-JIE QIN ${ }^{1}$, WEI MA ${ }^{2}$, SHAN-XI LIU ${ }^{3}$, GUANG-XIAO YANG ${ }^{4}$, QUAN-YING WANG ${ }^{4}$ and XIN-HAN ZHAO ${ }^{1}$ \\ Departments of ${ }^{1}$ Oncology, ${ }^{2}$ Orthopaedics, ${ }^{3}$ Hematology, First Affiliated Hospital of Medical College of \\ Xi'an Jiaotong University; ${ }^{4}$ Xi'an Huaguang Bio-engineering Co., Shaanxi 710061, P.R. China
}

Received April 12, 2010; Accepted July 12, 2010

DOI: $10.3892 / \mathrm{mmr} .2010 .343$

\begin{abstract}
Prostate apoptosis response-4 (Par-4) is a tumorsuppressor protein that induces apoptosis in cancer cells, but not in normal cells. The cancer-specific pro-apoptotic action of Par-4 is encoded in its centrally located SAC domain. In this study, to further enhance the anti-cancer effect of Par-4 in order to overcome the limitations of peptide therapy, a recombinant adeno-associated virus was constructed using the following strategies: the secretory expression of therapeutic peptide, a HA2TAT-mediated cytosolic delivery technique, and an adenoassociated virus gene transfer system. To test the hypothesis that Par-4 has an additive bystander effect as an anti-cancer therapy, we designed a secretory protein by adding a secretory signal peptide NT4(Si) to the Par-4 SAC-HA2TAT peptide gene sequence [NT4(Si)-Par-4 SAC-HA2TAT]. The results indicated that, compared to the normal NIH3T3 cell line, AAV-NT4(Si)Par-4 SAC-HA2TAT significantly suppressed cell growth and induced rapid cell death in HepG2 cells in a time-dependent manner through successful gene transfer and secretory expression of therapeutic peptide at $48 \mathrm{~h}$ post-transfection. In addition, the secretory properties of Par-4 may greatly increase its effectiveness in cancer therapy when delivered in vivo.
\end{abstract}

\section{Introduction}

The prostate apoptosis response 4 gene (par-4 or PAWR) was identified in a differential screen for pro-apoptotic genes in prostate carcinoma cell lines (1). The Par-4 gene maps to human chromosome $12 \mathrm{q} 21$, a region frequently deleted in certain malignancies (2), and encodes a $38 \mathrm{kDa}$ protein containing two nuclear localization signal (NLS) domains (3) and a leucine zipper domain in the $\mathrm{COOH}$-terminal region,

Correspondence to: Professor Xinhan Zhao, Department of Oncology, First Affiliated Hospital of Medical College of Xi'an Jiaotong University, No. 277, Yanta West Road, Xi'an, Shaanxi 710061, P.R. China

E-mail: zhaoxinhan@mail.xjtu.edu.cn

Key words: Par-4 SAC, HA2TAT, secretory expression which interacts with a variety of proteins, including the atypical protein kinases $\zeta \mathrm{PKC}, \lambda \mathrm{PKC}$ and the Wilms' tumor suppressor protein WT1 (4-7).

Par-4 is a tumor-suppressor protein that selectively induces apoptosis in cancer cells, but not in normal/immortalized cells, and is widely expressed in diverse normal and cancerous cell types and tissues (8). In normal or immortalized cells, Par-4 does not enter the nucleus and is unable to induce apoptosis $(9,10)$. The Par-4 SAC domain, a 59-amino-acid (137-195: GKSSGPSARKGKGQIEKRKLREKRRSTGVVNI PAAECLDEYEDDEAGQKERKREDAITQ) core domain that includes NLS2, is necessary and sufficient to induce Fas pathway activation, the inhibition of NF- $\mathrm{KB}$ activity, and apoptosis (9). An essential feature of anti-cancer strategies is selective action against cancer cells, with little or no damage inflicted on normal cells. Therefore, the SAC domain, which represents the minimal functional pro-apoptotic unit of Par-4, is an ideal candidate for anti-cancer therapy $(8,9)$.

One of the many hurdles facing gene therapists is the requirement to optimize gene delivery systems in such a way that both efficient and selective transgene production is achieved in vivo within a chosen cell or tissue (11). Vectors based on recombinant adeno-associated virus (rAAV) offer a number of attractive features and are emerging as promising gene transfer vehicles for many in vivo applications. Unfortunately, the utility of peptides directly injected into solid tumors is limited by their short biological half-life, degradation, difficulty in reaching the effective dose and high cost. We therefore constructed a novel recombinant adeno-associated virus containing a secretory expression cassette [signal peptide sequence and pro region of neurotrophin 4(NT4) fused to the Par-4 SAC-HA2TAT peptide gene sequence] to explore whether Par-4 SAC-HA2TAT fusion gene transfer and secretory expression is a promising cancer gene therapy approach. The anti-cancer effect of this fusion peptide derived from Par-4 SAC would be reinforced if transfected tumor cells themselves secreted this peptide, interacting with other surrounding untransfected tumor cells. Co-treatment with the fused NT4(Si)-Par-4 SAC-HA2TAT peptide may be a promising approach for validating intracellular targets of effective peptide-based anti-cancer drugs, and thus may have an additive bystander effect as an anti-cancer therapy. 


\section{Materials and methods}

Cell lines and cell culture. The human hepatocellular carcinoma cell line HepG2 was cultured in RPMI-1640 containing $10 \%$ heat inactivated fetal bovine serum (FBS; Gibco BRL). Human embryonic kidney 293 cells and NIH3T3 cells were maintained in Dulbecco's modified Eagle's medium (DMEM; Gibco BRL) supplemented with $10 \% \mathrm{FBS}, 100 \mathrm{U} / \mathrm{ml}$ penicillin and $100 \mu \mathrm{g} / \mathrm{ml}$ streptomycin. The cell lines were purchased from the American Type Culture Collection and maintained at $37^{\circ} \mathrm{C}$ in a $5 \%$ carbon dioxide humidified incubator. Subsequent experiments were performed using cells harvested during the exponential growth phase.

Primers design and synthesis. The Par-4 SAC DNA fragments with two restriction enzymes, NaeI and KpnI (underlined), at two extreme ends were synthesized by Beijing Sun Biotechnology Co. Primers were designated according to the published sequence of the Par-4 SAC gene in GenBank (NM_002583). In this study, four primers were designated, two forward and two reverse. The primers sequences were as follows: forward primer F1, 5'-gGCCGGCGTGGGATG GGCAAGAGCTCGGGCCCCAGTGCCAGGAAAGG CAAGGGGCAG-3', and reverse primer R1, 5'-GACCAC GCCGGTGGAGCGCCGCTTCTCCCGCAGCTTCCT CTTCTCGATCTGCCCCTTG-3'; forward primer F2, 5'-GGCGTGGTCAACATCCCTGCCGCAGAGTGCTTA GATGAGTACGAAGATGATGAAGCAGG-3', and reverse primer R2，5'-gGGTACCTTGTGTAATTGCATCTTCTCG TTTCCGCTCTTTCTGCCCTGCTTCAT-3'. Primer F1 contains a complementary overlap region with primer R1 (bold), as do primers F2 with R2 and F2 with R1. The Par-4 SAC gene was spliced by overlap extension. Amplification was carried out in a $100-\mu 1$ volume PCR mix containing $10 \mu \mathrm{l}$ PCR buffer 10X, $2 \mu \mathrm{l}$ dNTPs, $2 \mu \mathrm{l}$ of each PCR primer (F1, F2 and R1, R2) (50 pmol/ $\mu \mathrm{l}), 1 \mu \mathrm{l} \mathrm{Taq}$ Polymerase (Promega) and $79 \mu 1 \mathrm{H}_{2} \mathrm{O}$ under the following thermocycling conditions: initial denaturation at $94^{\circ} \mathrm{C}$ for $5 \mathrm{~min}$, followed by 30 cycles of amplification at $94^{\circ} \mathrm{C}, 60 \mathrm{sec}$ of denaturation; $37^{\circ} \mathrm{C}, 60 \mathrm{sec}$ of annealing; $72^{\circ} \mathrm{C}, 80 \mathrm{sec}$ of extension and a final extension for $5 \min$ at $72^{\circ} \mathrm{C}$. The four primers produced a 199-bp fragment that was used in the subsequent experiment.

Cloning and nucleotide sequencing of Par-4 SAC cDNA. After identification (Par-4 SAC) by digestion with the restriction enzymes and DNA sequencing, the purified PCR products were subcloned into pGEM-T-easy vector (Promega). Cloning procedures were performed according to the manufacturer's instructions, and the resulting plasmids were examined by restriction enzyme digestion and agarose gel electrophoresis. DNA sequencing was performed by Shanghai Sangon Biological Engineering Technology and Services (Shanghai, P.R. China). Sequence analysis was carried out using Applied Biosystems 310 DNA analysis instruments.

Plasmid construction. The HA2TAT DNA fragment with two restriction enzymes, $K p n I$ and $X h o I$, at two extreme ends was synthesized by Huaguang Bio-engineering Co. according to the method described by Sugita et al (12) and GenBank. The HA2TAT fragment was then subcloned into
pGEM-T-easy vector. After identification by digestion with restriction enzymes and DNA sequencing, the Par-4 SAC and HA2TAT fragments were inserted into the pUC19/ NT4(Si) vector (Huaguang Bio-engineering Co.), pre-digested with EcoRI and NaeI, and resulted in pUC19/NT4(Si)-Par-4 SAC-HA2TAT. The signal peptide sequence and pro region of NT4 was polymerase chain reaction (PCR)-cloned from human genomic DNA and subcloned into the EcoRI/NaeI site of the pUC19 vector. After digestion with EcoRI and XhoI, the NT4(Si)-Par-4 SAC-HA2TAT fragment from the pUC19/ NT4(Si)-Par-4 SAC-HA2TAT plasmid was inserted into multiple cloning sites of the expression plasmid pSSHG-CMV. The resulting plasmid was named pSSHG-CMV/NT4(Si)Par-4 SAC-HA2TAT. The recombinant plasmids were transformed into the appropriate E. coli DH5 (Invitrogen) competent cells according to the manufacturer's protocol. Plasmid DNA was confirmed by restriction enzyme digestion and DNA sequencing.

To generate a control expression plasmid containing green fluorescent protein, the EGFP gene was amplified from EGFP-C2 plasmid (Huaguang Bio-engineering Co.) by PCR. Primer sequences were as follows: forward primer, 5'-CGGGATCCATGGTGAGCAAGGGCGAGG-3' and reverse primer, 5'-CGCTCGAGTCAAGTCCGGCCGGACTTGTAC-3'. After verification by DNA sequencing, the resulting PCR fragment was digested with BamHI and $\mathrm{XhoI}$ (underlined) and inserted into the pSSHG-CMV plasmid.

Virus production and titer determination. The three-plasmidbased adeno-associated virus (AAV) expression system was purchased from Invitrogen. Briefly, $20 \mu \mathrm{g}$ of pFG140 plasmid, $10 \mu \mathrm{g}$ of pAAV/Ad plasmid and $10 \mu \mathrm{g}$ of pSSHG-CMV/ NT4(Si)-Par-4 SAC-HA2TAT or pSSHG-CMV/EGFP were co-transfected into 293 cells using the calcium phosphate co-precipitation method. Conditioned medium was harvested at $72 \mathrm{~h}$ after transfection and filtered through $0.45-\mu \mathrm{m}$ filters. Concentrated viral stocks were prepared by ultracentrifugation of $3 \mathrm{ml}$ conditioned medium at $40,000 \mathrm{x} \mathrm{g}$ at $4^{\circ} \mathrm{C}$ for $1.5 \mathrm{~h}$ in an SW41 rotor (Beckman). Then, the pellet was resuspended in $30 \mu \mathrm{l}$ of complete medium and frozen at $-80^{\circ} \mathrm{C}$. The resulting recombinant AAV was named AAV-NT4(Si)Par-4 SAC-HA2TAT or AAV-EGFP. The recombinant AAV viral stock was titrated by dot blot.

$A A V$ mediated gene transfer and expression in HepG2 cells. HepG2 cells were seeded into 6-well plates at a density of $1 \times 10^{6}$ cells/well. Cells were allowed to adhere for $24 \mathrm{~h}$ and then infected with AAV-EGFP at multiplicities of infection (MOI) of 4 transducting units (TU)/cell. The EGFP-positive cells were observed and calculated under a fluorescence microscope at $48 \mathrm{~h}$ post-infection.

The expression of NT4(Si)-Par-4 SAC-HA2TAT mRNA was detected by reverse transcription (RT)-PCR. HepG2 cells were seeded into $100-\mathrm{ml}$ culture flasks at a density of $2 \times 10^{6}$ cells/flask and infected with AAV-NT4(Si)-Par-4 SAC-HA2TAT (MOI, 4 TU/cell). After 48 h, total RNA was extracted using TRIzol reagent (Gibco, Los Angeles, CA, USA). The RT step was carried out for $60 \mathrm{~min}$ at $37^{\circ} \mathrm{C}$ with $5 \mu \mathrm{g}$ of total RNA that had been treated with RNasin and AMV reverse transcriptase (Invitrogen) in the presence of random 
A

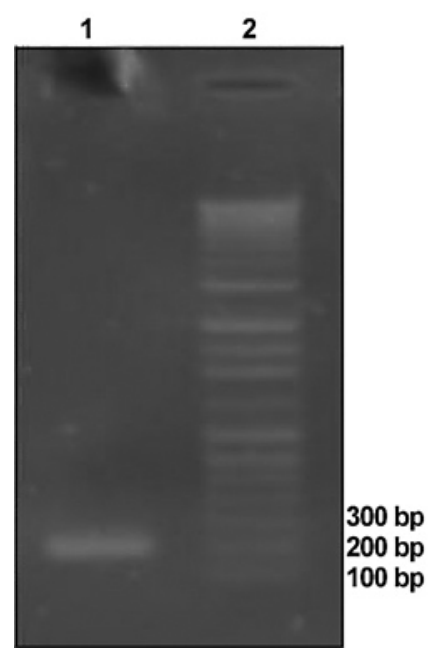

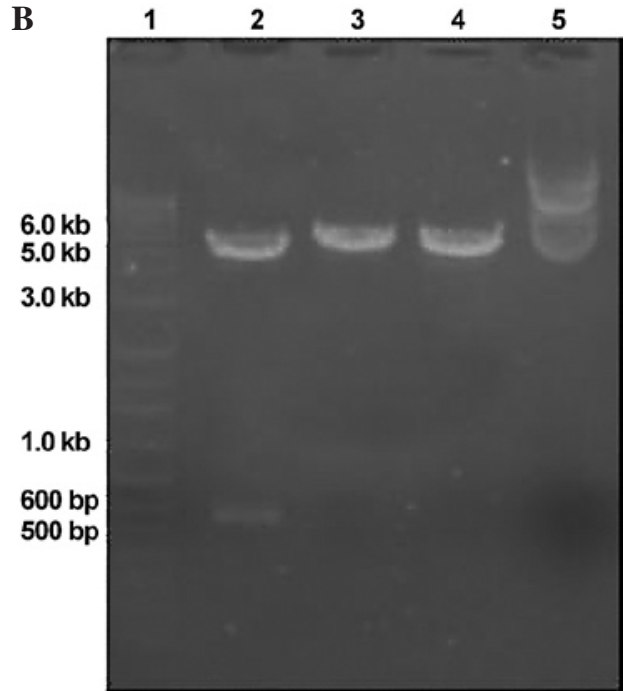

Figure 1. Identification of Par-4 SAC DNA and adeno-associated virus expression vectors. (A) Restriction enzyme analysis of PCR products of Par-4 SAC. The DNA fragment was determined by agarose gel electrophoresis. Lane 1, Par-4 SAC DNA; lane 2, 12 kb DNA marker. (B) pSSHG-CMV/NT4(Si)-Par-4 SAC-HA2TAT identified by restricted digestion. Lane 1, DNA Ladder (12 kb); lane 2, EcoRI and XhoI enzyme restrictive digestion; lanes 3 and 4, pSSHG-CMV/ NT4(Si)-Par-4 SAC-HA2TAT plasmid digested with EcoRI only; lane 5, negative control of pSSHG-CMV.

primers. PCR was carried out in $100-\mu 1$ reaction volumes containing $10 \mu \mathrm{l}(2.5 \mathrm{ng}) \mathrm{cDNA}, 2 \mu \mathrm{l} 10 \mathrm{mmol} / \mathrm{l} \mathrm{dNTPs}$, 1 unit of Taq DNA polymerase, $10 \mu \mathrm{l}$ of $10 \mathrm{X}$ Taq buffer and $1 \mu 150$ pmol of NT4(Si)-Par-4 SAC-HA2TAT primers (forward primer, 5'-ATGCTCCCTCTCCCCTCATGC-3'; reverse primer, 5'-TCATCTTCGTCGCTGTCTCCGC-3'). RT-PCR procedures and conditions were performed as follows: initial denaturation at $94^{\circ} \mathrm{C}$ for $3 \mathrm{~min}$, followed by 30 cycles at $94^{\circ} \mathrm{C}, 1 \mathrm{~min}$ of denaturation; $53^{\circ} \mathrm{C}, 1 \mathrm{~min}$ of annealing; $72^{\circ} \mathrm{C}, 90 \mathrm{sec}$ of extension and a final extension for $5 \mathrm{~min}$ at $72^{\circ} \mathrm{C}$ in a thermal cycler. The RT-PCR products were electrophoresed on $2 \%$ agarose gel and documented by photography.

Morphological analysis. Exponentially growing cells (HepG2 and NIH3T3) were incubated at a density of $1 \times 10^{5}$ cells/ well in 24-well plates and infected with AAV-NT4(Si)-Par-4 SAC-HA2TAT or AAV-EGFP. Untreated cells served as the control. Cells were harvested at three time points $(24,48$ or $72 \mathrm{~h}$ ) after transfection. For morphological analysis, cells were observed under a phase-contrast microscope (Olympus, Japan) at various intervals.

MTT assay. The effect of AAV-NT4(Si)-Par-4 SAC-HA2TAT on cancer cell viability was determined by 3-[4,5-dimethylthiazol-2yl]-2,5 diphenyl tetrazolium bromide (MTT) dye reduction assay, which measures mitochondrial respiratory function. Exponentially growing cells (HepG2 and NIH3T3) were plated in 96-well plates and grown for $24 \mathrm{~h}$, then transfected with AAV-NT4(Si)-Par-4 SAC-HA2TAT or AAV-EGFP. The culture medium was replaced with fresh medium after $12 \mathrm{~h}$. At various time points (24, 48 and $72 \mathrm{~h}$ ) after adeno-associated virus infection, the MTT assay was performed according to the manufacturer's instructions. The optical density (OD) was determined by eluting the blue crystals with DMSO, and the absorbance was measured at $490 \mathrm{~nm}$ by a microplate reader (Labsystems, Finland). All MTT assays were performed in triplicate.
Flow cytometric analysis. At $48 \mathrm{~h}$ after infection with AAV-NT4(Si)-Par-4 SAC-HA2TAT or AAV-EGFP, both floating and attached HepG2 and NIH3T3 cells were collected from each sample. The cells were fixed in $70 \%$ ethanol, treated with $0.1 \mathrm{~g} / 1$ RNase A, washed twice with PBS and stained with $100 \mathrm{mg} / \mathrm{l}$ propidium iodide. DNA content was analyzed by flow cytometry (Facscalibur BD, San Jose, CA, USA) and the fraction of cells with sub-G1 DNA content was quantified. At least three independent experiments were performed. The percentage of cells in the subdiploid region was considered an index of apoptosis. Cells treated with AAV-EGFP were run in parallel and served as the positive control, while untreated cells served as the negative control.

Protein secretion and activity test. The bystander effect was measured, as well as the killing effect of the reactive supernatant in tumor cells treated with AAV-NT4(Si)-Par-4 SAC-HA2TAT. HepG 2 cells were cultured in a 6-well plate and then transfected with the AAV-NT4(Si)-Par-4 SAC-HA2TAT and AAV-EGFP plasmids. After a 6-h incubation, the cells were washed with fresh culture medium and cultured for an additional $96 \mathrm{~h}$. The culture supernatants were then respectively collected and added to the monolayers of HepG2 cells grown in 24-well plates. These cells had also been washed with PBS before the addition of the supernatants. At various times post co-culture, the MTT assay and morphological changes were analyzed. Assays were performed at least in triplicate.

Statistical analysis. Data are presented as the mean \pm SD (standard deviation of the mean). Statistical comparison between the treated and control groups was performed by the two-tailed Student's t-test. Statistical analysis was performed using SPSS software (version 12.0; SPSS, Chicago, IL, USA). Differences were considered to be statistically significant at a $\mathrm{P}$-value of $<0.05$. 

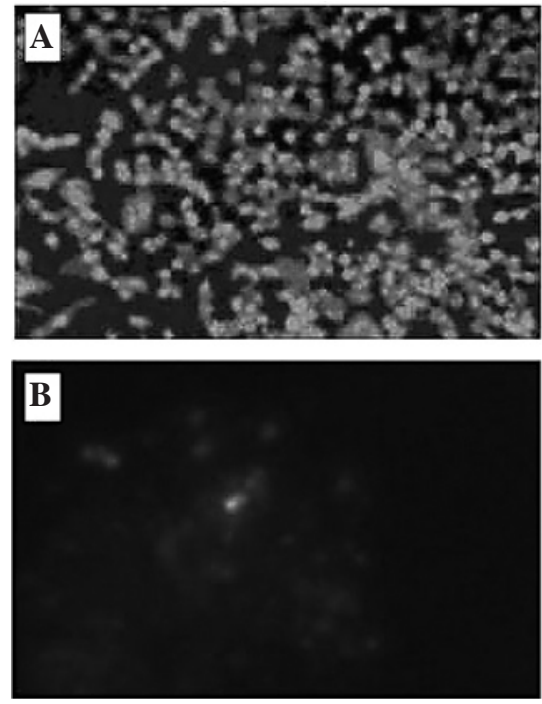

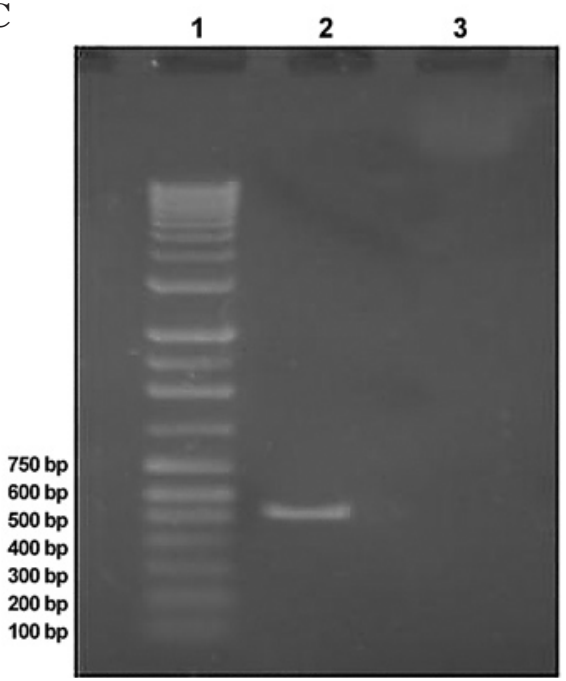

Figure 2. Expression of NT4(Si)-Par-4 SAC-HA2TAT and EGFP in HepG2 cells. (A) Fluorescent microscopic analysis showing EGFP expression in HepG2 cells at $48 \mathrm{~h}$ after infection. The number of EGFP-positive cells was observed under a x400 original magnification. (B) Control cells. (C) Agarose gel electrophoresis of RT-PCR products from HepG2 cells infected by AAV-NT4(Si)-Par-4 SAC-HA2TAT at $48 \mathrm{~h}$ after infection. Lane 1, $12 \mathrm{~kb}$ DNA ladder; lane 2, RT-PCR product from cells infected with AAV-NT4 (Si)-Par-4 SAC-HA2TAT; lane 3, negative control (infected with AAV-EGFP).

\section{Results}

Construction of an AAV vector expressing NT4(Si)-Par-4 SAC-HA2TAT. Adeno-associated virus vectors are generated by transfecting producer cells with a plasmid containing a recombinant AAV (rAAV) genome composed of foreign DNA flanked by AAV ITRs and a construct expressing in trans the viral rep and cap genes. In the presence of Ad helper functions, the rAAV genome is rescued from the plasmid backbone, replicated and packaged into AAV capsids. The AAV expression vector $\mathrm{pSSHG-CMV}$ contains the elements required to allow packaging of the expression construct into virions.

DNA sequencing of the Par-4 SAC fragment confirmed that the result was identical to that of Par-4 SAC published in GenBank. The total DNA length of NT4(Si)-Par-4 SAC-HA2TAT is $547 \mathrm{bp}$, including a $255 \mathrm{bp}$ DNA fragment of the signal peptide sequence and pro region of NT4 with two restriction sites (EcoRI and NaeI), and a 292 bp DNA fragment of Par-4 SAC-HA2TAT with NaeI and XhoI. Analysis of the vector pSSHG-CMV/NT4(Si)-Par-4 SAC-HA2TAT by restriction enzyme digestion (EcoRI and XhoI) verified that the NT4(Si)-Par-4 SAC-HA2TAT secretory expression cassette was correctly inserted into pSSHG-CMV (Fig. 1).

Expression of NT4(Si)-Par-4 SAC-HA2TAT delivered by adeno-associated virus vector. Biophysical probes, such as fluorophores, are useful for investigating the process of AAV infection. Enhanced green fluorescent protein (EGFP) is an attractive option because of its high labeling specificity and ease of use (13). These ubiquitous probes have been extremely useful as reporters for gene expression studies in cultured cells and tissues, as well as living animals (14).

Green fluorescent staining in HepG2 cells was observed using an inverted fluorescence microscope. EGFP-positive cells were counted. When cells were infected with AAV-EGFP at a MOI of $4 \mathrm{TU} /$ cell, $>80 \%$ of cells were EGFP-positive at $48 \mathrm{~h}$ post-transfection (Fig. $2 \mathrm{~A}$ ).
To determine whether the NT4(Si)-Par-4 SAC-HA2TAT cDNA construct generated was expressed in vivo, the expression of NT4(Si)-Par-4 SAC-HA2TAT in HepG2 cells was detected by RT-PCR. The HepG2 cell line was transfected with the AAV-NT4(Si)-Par-4 SAC-HA2TAT plasmid. Analysis by RT-PCR revealed that NT4(Si)-Par-4 SAC-HA2TAT was expressed at $48 \mathrm{~h}$ after transfection. As expected, an 547-bp transcription product was detected in AAV-NT4(Si)-Par-4 SAC-HA2TAT-infected cells, but not in the control cells (Fig. 2C), which proved that the process of adeno-associated virus construction was successful and that the recombinant adeno-associated virus possessed the ability to efficiently infect and express the gene in target cells.

Morphological changes in HepG2 cells after transfection with AAV-NT4(Si)-Par-4 SAC-HA2TAT. Morphologically, rapid cell shrinkage, increases in cell granularity and chromatin condensation are the most notable changes associated with apoptosis. These changes allow populations of apoptotic and normal cells to be distinguished using light microscopy.

In this study, significant morphological changes of HepG2 cells showed cell apoptosis in cells treated with AAV-NT4(Si)-Par-4 SAC-HA2TAT vs. AAV/EGFP and untreated cells (Fig. 3C-F). NIH3T3 cells treated with AAV-NT4(Si)-Par-4 SAC-HA2TAT and PBS showed no marked differences in morphology (Fig. 3A and B). These results demonstrated that treatment with AAV-NT4(Si)-Par-4 SAC-HA2TAT effectively elicits cell killing in cultured human liver cancer cells, but not in normal human fibroblasts.

Cytotoxicity of AAV-NT4(Si)-Par-4 SAC-HA2TAT in HepG2 cells. Cytotoxicity effects on the HepG2 cells after infection with AAV-NT4(Si)-Par-4 SAC-HA2TAT were determined by the MTT assay, which is based on the ability of mitochondrial dehydrogenases in viable cells to reduce MTT, a soluble yellow tetrazolium salt, to blue formazan crystals. 

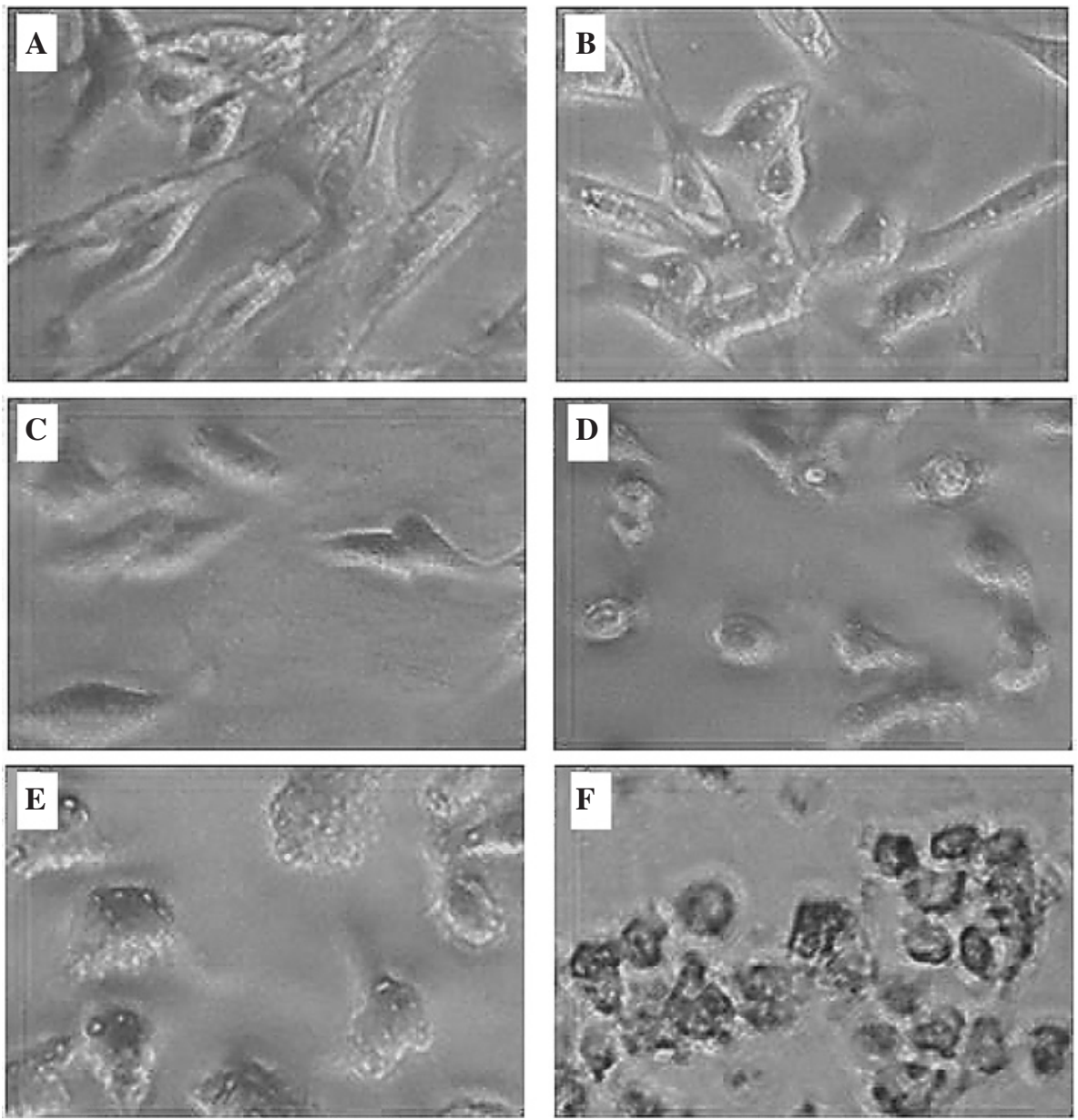

Figure 3. Morphological changes in NIH3T3 and HepG2 cells after treatment with AAV-NT4(Si)-Par-4 SAC-HA2TAT and AAV-EGFP for 24 and 48 h determined by phase contrast microscopy (x400). (A) Untreated control NIH3T3 cells. (B) NIH3T3 cells 48 h after transfection with AAV-NT4(Si)-Par-4 SAC-HA2TAT. (C) Untreated control HepG2 cells. (D) HepG2 cells 48 h after infection with AAV-EGFP. (E) HepG2 cells 24 h after transfection with AAV-NT4(Si)-Par-4 SAC-HA2TAT. (F) HepG2 cells 48 h after transfection with AAV-NT4(Si)-Par-4 SAC-HA2TAT. Morphologically, rapid cell shrinkage, increases in cell granularity and chromatin condensation are the most notable changes associated with apoptosis. Morphological changes in HepG2 cells $24 \mathrm{~h}$ (E) and $48 \mathrm{~h}$ (F) after transfection with AAV-NT4 (Si)-Par-4 SAC-HA2TAT exhibited the characteristics of apoptosis, while the control cells were unaffected. NIH3T3 cells treated with AAV-NT4(Si)-Par-4SAC-HA2TAT (B) showed no marked differences compared to untreated cells (A), nor did HepG2 cells treated with AAV-EGFP (D) compared to untreated cells (C).

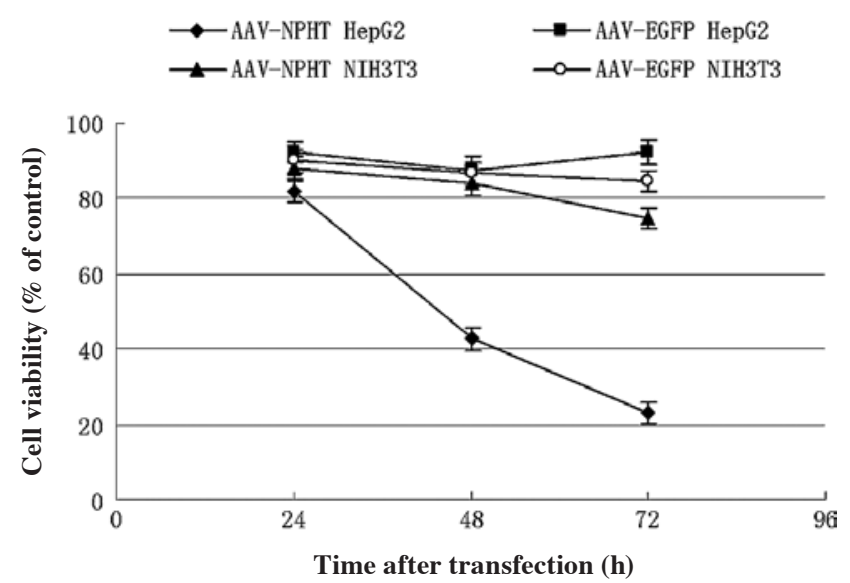

Figure 4. Effect of AAV-NT4(Si)-Par-4 SAC-HA2TAT on the proliferation and viability of HepG2 and NIH3T3 cells determined by the MTT assay. Data are represented as the mean \pm SD of at least three independent analyses. The HepG2 cancer cell line and NIH3T3 cells were infected with AAVNPHT [AAV-NT4(Si)-Par-4 SAC-HA2TAT] or AAV-EGFP, respectively. At various time points $(24,48$ and $72 \mathrm{~h})$ after rAAV infection, the MTT assay was conducted to determine the effect of AAV-NT4(Si)-Par-4 SAC-HA2TAT on cancer cell viability.
After transfection, cell viability was determined at various times by the MTT dye reduction assay. Expression of recombinant protein slightly decreased the viability of HepG2 cells at $24 \mathrm{~h}$ post-transfection. The same was true in NIH3T3 cells (Fig. 4). At $48 \mathrm{~h}$ post-transfection, the viability of NIH3T3 cells was only slightly decreased compared to that at $24 \mathrm{~h}$. However, the viability of HepG2 cells was significantly decreased at $48 \mathrm{~h}$ post-transfection. The presence of recombinant Par-4 SAC caused a decrease in HepG2 cell viability to $<50 \%$ at $48 \mathrm{~h}$ post-transfection.

The cell viability study showed a significant difference in cell killing between HepG2 and NIH3T3 cells. The MTT assay demonstrated that, compared to the control group transfected with AAV-EGFP, AAV-NT4(Si)-Par-4 SAC-HA2TAT undoubtedly had a cytotoxic effect on the cancer cells (Fig. 4). Moreover, the cell viability of the HepG2 cells was gradually decreased over time after infection with AAV-NT4(Si)-Par-4 SAC-HA2TAT, which indicates that the cytotoxicity of NT4(Si)Par-4 SAC-HA2TAT operated in time-dependent manner. Additionally, the expression of NT4(Si)-Par-4 SAC-HA2TAT appears to have no obvious cytotoxic effect on NIH3T3 cells. 
A

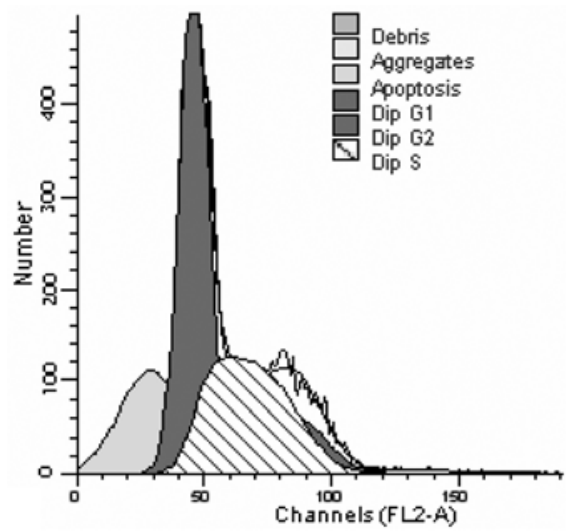

B

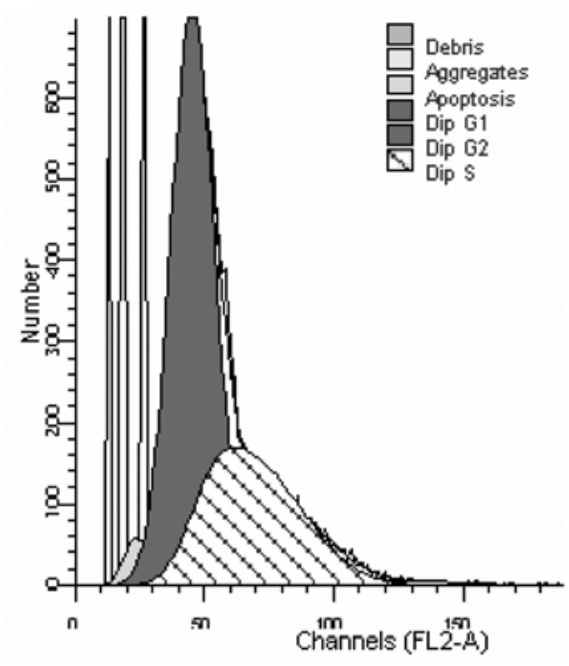

D

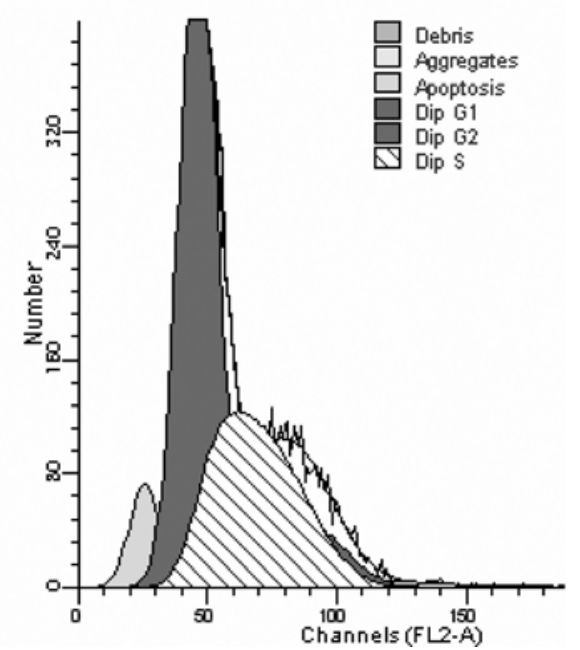

C

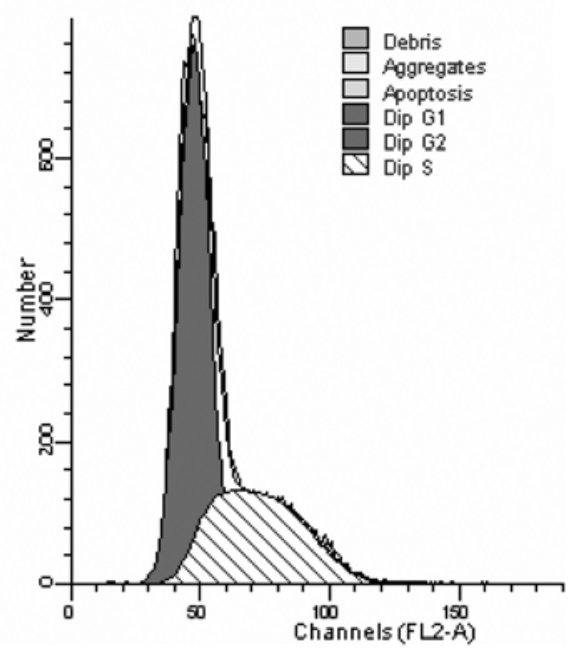

$\mathbf{E}$

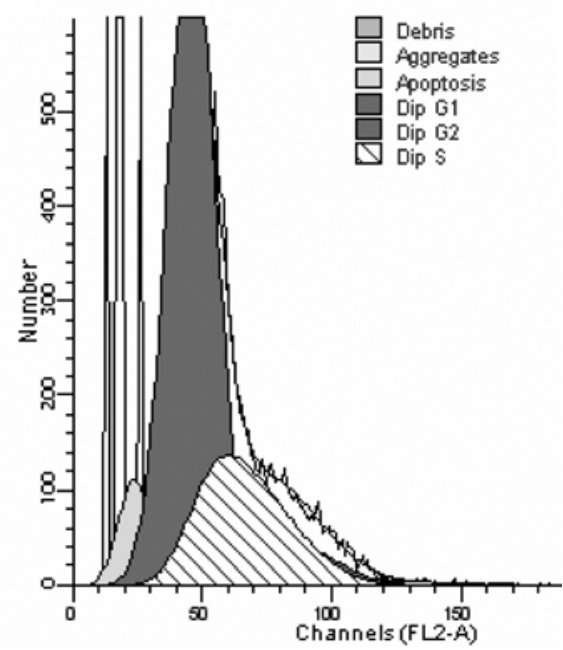

Figure 5. Analysis of nuclear DNA contents by propidium iodide staining combined with flow cytometry. (A) HepG2 cells transfected with AAV-NT4(Si)Par-4 SAC-HA2TAT. (B) HepG2 cells infected with AAV-EGFP. (C) Untreated HepG2 cells. (D) NIH3T3 cells transfected with AAV-NT4(Si)-Par-4 SAC-HA2TAT. (E) NIH3T3 cells infected with AAV-EGFP. At $30 \mathrm{~h}$ after transfection with AAV-NT4(Si)-Par-4 SAC-HA2TAT, the ratio of apoptotic cells in the HepG2 and NIH3T3 cell lines was $18.50 \pm 4.23$ and $6.33 \pm 1.02 \%$, respectively, and in the HepG2 and NIH3T3 cell lines treated with AAV-EGFP, $2.70 \pm 0.51$ and $6.22 \pm 1.11 \%$, respectively.

Apoptosis and cell cycle analysis. Examination of nuclear DNA contents by flow cytometry revealed that AAV-NT4(Si)Par-4 SAC-HA2TAT induced cell apoptosis at $48 \mathrm{~h}$ post-transfection (Fig. 5).

Flow cytometry indicated that the ratios of apoptotic cells in the HepG2 cell line transfected with AAV-NT4(Si)-Par-4
SAC-HA2TAT or AAV-EGFP were $18.50 \pm 4.23$ and $2.70 \pm 0.51$, respectively, and in untreated cells, $0.00 \pm 0.01 \%$. There was a significant difference between these results $(\mathrm{P}<0.05)$. The ratios of apoptotic cells in the HepG2 and NIH3T3 cell lines after infection with AAV-NT4(Si)-Par-4 SAC-HA2TAT were $18.50 \pm 4.23$ and $6.33 \pm 1.02 \%$, respectively, and the 

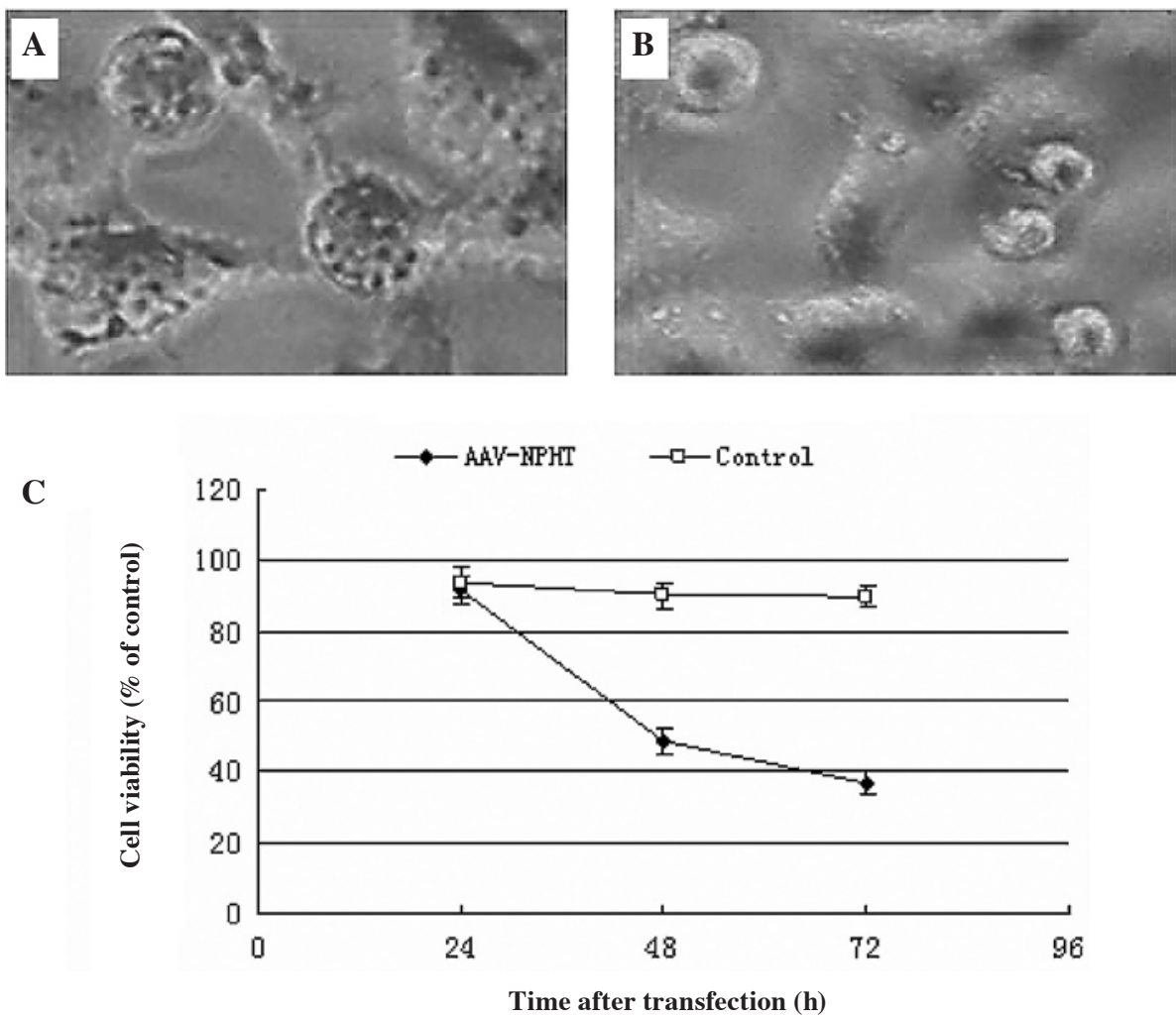

Figure 6. (A and B) Phase-contrast photomicrographs of the bystander anti-tumor effect (x400). Morphological apoptotic changes in HepG2 cells induced by secreted Par-4 SAC-HA2TAT (A) showed obvious difference compared to control cells (B). (C) Cell viability determined by MTT dye reduction assay after co-culture with secreted Par-4 SAC-HA2TAT.

ratio of apoptosis in HepG2 cells was significantly higher than that in NIH3T3 cells $(6.22 \pm 1.11 \%)$ after infection with AAV-EGFP $(\mathrm{P}<0.05)$. This indicates that AAV-NT4(Si)-Par-4 SAC-HA2TAT has a greater ability to induce apoptosis in human liver cancer cells than in normal cells.

In addition, the HepG 2 cells displayed a decrease in the G1 peak from $65.36 \pm 6.50$ to $52.40 \pm 4.70 \%$ and an increase in the $\mathrm{G} 2$ peak from $0.31 \pm 0.23$ to $11.17 \pm 3.02 \%$ compared to the control cells after infection for $48 \mathrm{~h}$. The sub-G1 peak was detected, which suggests that cell cycle regulation may have been influenced by AAV and the expression of NT4(Si)-Par-4 SAC-HA2TAT.

Effect of secreted Par-4 SAC-HA2TAT on HepG2 cells. The presence of NT4(Si) enables Par-4 SAC-HA2TAT to be secreted outside the transfected cells and to re-enter adjacent un-transfected HepG2 cells, potentially increasing the efficacy of Par-4 SAC-HA2TAT used as cancer therapy. To determine the feasibility of its use, the recombinant construct was used to transfect HepG2 cells and the culture supernatant was collected. HepG2 cells were then co-cultured with this supernatant. At various time points post co-culture, significant apoptotic morphological changes in HepG2 cells (but not in control cells) were observed by light microscopy, and were indicative of apoptosis (Fig. 6A and B). It was also possible to visualize the bystander effect of rAAV by the MTT dye reduction assay. As shown in Fig. 6C, the recombinant protein NT4(Si)-Par-4 SAC-HA2TAT slightly decreased the cell viability of HepG 2 cells at $24 \mathrm{~h}$ post co-culture. By contrast, at $48 \mathrm{~h}$ post co-culture, the viability of HepG2 cells was significantly decreased compared to that of the control cells. The recombinant Par-4 SAC protein decreased HepG2 cell viability to $<50 \%$ at $72 \mathrm{~h}$ post-transfection. Therefore, NT4(Si)-Par-4 SAC-HA2TAT is effective in killing both HepG2 cells and surrounding tumor cells.

\section{Discussion}

Hepatocellular carcinoma (HCC) is one of the most common malignant tumors worldwide. Its global incidence continues to increase (15), so effective systemic therapy for advanced $\mathrm{HCC}$ is urgently required. Apart from conventional systemic chemotherapy, targeted therapy potentially provides another treatment modality for advanced-stage HCC patients. The toxicity of targeted therapy is generally much lower than that of conventional cytotoxic agents due to its improved selectivity against cancer cells (16).

Since Par-4 SAC does not cause the apoptosis of nontumor cells, replenishment of Par-4 SAC levels or reactivation of the pathways of Par-4 SAC-mediated apoptosis represent promising targets for cancer treatment and gene therapy $(9,17)$. The ability of Par- 4 SAC to selectively induce apoptosis in transformed cells makes it attractive as an apoptosis-inducing agent for cancer therapy as compared to other pro-apoptotic proteins. In this study, we constructed a novel recombinant adeno-associated virus [AAV-NT4(Si)-Par-4 SAC-HA2TAT]. The aim of this study was to evaluate the anti-proliferative and cytotoxic properties of NT4(Si)-Par-4 SAC-HA2TAT in a human HepG2 cell line and to explore whether this protein has potential use in cancer gene therapy. 
Since the therapeutic targets of cancer generally exist inside the cell, it is necessary to establish drug (gene/peptide) delivery methods that transfer macromolecules, such as therapeutic proteins or peptide-based drugs, across the cellular membrane. Protein transduction domains (PTDs) have been extensively utilized for intracellular delivery in vitro and in vivo (18-20). The best-known example of a PTD is the TAT-mediated delivery of peptide-blockers. TAT peptides are useful carriers for delivering biologic molecules into the cell for the functional analysis of intracellular disease-related proteins and the treatment of refractory diseases (21).

Meanwhile, most of the treated peptides are entrapped in the macropinosomes, limiting the biologic function of the cargo (for example, the Par-4 SAC). With this in mind, we constructed the recombinant Par-4 SAC-HA2TAT, with the aim of enhancing the endosome-escape efficiency of the TAT-cargos using the $\mathrm{N}$-terminal 20 amino acid peptide of the influenza virus hemagglutinin protein (HA2). Several recent studies have shown that TAT fused with HA2 (HA2TAT GLFEAIEGFIENGWEGMIDGWYGYGRKKRRQRRR) markedly enhances the disruption of macropinosomes. This HA2TAT-mediated cytosolic delivery technique leads to the enhanced cytotoxicity of TAT-fused anti-cancer peptides $(12,22)$.

One of the obstacles facing gene therapy for malignant tumors is low transgene efficiency, as it is difficult to transfer target genes into each tumor cell. The focus of our studies is how to enhance the anti-cancer effect of these genes and simultaneously how to overcome the disadvantages of targeted therapy. Previous investigations have attempted to circumvent this limitation by exploiting what are called bystander effects, based on the transfer of vector transgene products from infected to uninfected cells. Bystander effects does not increase transgene efficiency; rather, they enhance cell killing ability (23). In this study, we employed two strategies: the application of an adenoassociated virus gene transfer strategy, and the application of a secretory expression strategy of the therapeutic peptide.

The development of vectors that enable efficient gene delivery and stable transgene expression is regarded as one of the main priorities in the field of gene therapy (24). Adenoassociated virus is a preferred vector for delivering therapeutic genes due to its safety profile and long-term gene expression in vivo (25). The recombinant AAV applied in this study may serve to improve gene delivery efficiency.

It is well known that signal peptides are located at the amino terminal of an amino acid sequence of a secretion protein. They play an important role in protein targeting and protein translocation in both prokaryotic and eukaryotic cells. This was our first attempt to utilize the NT4 signal peptide to mediate the entry of other therapeutic peptides into the secretory pathway (26). NT4 is processed in the constitutive secretory pathway, and is diffusely distributed within the cells and released, soon after being synthesized, in a manner that is not affected by cell depolarization (27). Analysis of the structure of NT4 shows that the human NT4 initation codon is followed by a signal peptide sequence, a pro region and an apparent dibasic cleavage site. This is followed by the sequence of the mature NT4. The signal peptide sequence and pro region of human NT4 is notably shorter (by $243 \mathrm{bp}$ ) than that of the other neurotrophins (26). Due to the insertion of the NT4 signal peptide, recombinant
Par-4 SAC was capable of being secreted from transfected cells and of re-entering adjacent untransfected HepG2 cells.

In this study, we successfully constructed rAAV containing the NT4(Si)-Par-4 SAC-HA2TAT fusion gene by in vitro recombinant DNA technology. RT-PCR detected the expression of NT4(Si)-Par-4 SAC-HA2TAT. By incorporating a synthetic signal peptide, -NT4(Si), we expected Par-4 SAC to be secreted from the transfected HepG2 cells as Par-4 SAC-HA2TAT fusion protein and to re-enter adjacent untransfected HepG2 cells, thus enabling NT4(Si)-Par-4 SAC-HA2TAT functional activity and efficiently inducing HepG2 cell apoptosis in a time-dependent manner. This construct acted as both a protein and a gene therapeutic agent, and increased the potency of Par-4 SAC in cancer therapy.

To investigate the effects of AAV-NT4(Si)-Par-4 SAC-HA2TAT, the MTT assay, morphological analysis and flow cytometric analysis were used. The results indicate that AAV-NT4(Si)-Par-4 SAC-HA2TAT induced apoptosis in HepG2 cells after infection for $48 \mathrm{~h}$, and that its cytotoxicity operated in a time-dependent manner. This is possibly related to the process of AAV infection, integration and the expression of the target gene, which gradually strengthened over time after AAV infection. In NIH3T3 cells, no induction of apoptosis above the background level was observed. HepG2 cell lines tested in our experiments showed a potent bystander effect, allowing for the destruction of a large percentage of nontransfected tumor cells in the presence of AAV-NT4(Si)-Par-4 SAC-HA2TAT-transfected tumor cells. This bystander effect greatly amplified the efficacy of NT4(Si)-Par-4 SAC-HA2TAT cancer gene therapy, while targeting only a fraction of the cells.

In conclusion, our results demonstrate that the growth of HepG2 cancer cells is significantly inhibited by AAV-NT4(Si)Par-4 SAC-HA2TAT. This study elucidated the mechanisms of the secretory expression of Par-4 SAC-HA2TAT peptide mediated by adeno-associated virus. This novel secretory characteristic increases the possibility of Par-4 SAC being used in cancer gene therapy. Our study thus contributes to the design of innovative cancer-selective therapeutics using Par-4 SAC. However, a large number of unanswered questions remain regarding the mechanisms and therapeutic usage of Par-4 SAC, and further studies are certainly required.

\section{Acknowledgements}

This study was supported by a grant from the Foundation for Science and Technology Development of Shaanxi Province (2005K09-G12). The authors gratefully thank V.M. Rangnekar for thoughtful suggestions, acknowledge the excellent technical assistance of Xinlu Wang and Yueping Li, and appreciate the technical assistance with flow cytometric analysis provided by the Medical College of Xi'an Jiaotong University.

\section{References}

1. Boosen M, Vetterkind S, Kubicek J, Scheidtmann KH, Illenberger S and Preuss U: Par-4 is an essential downstream target of DAP-like kinase (Dlk) in Dlk/Par-4-mediated apoptosis . Mol Biol Cell 20: 4010-4020, 2009.

2. Garcia-Cao I, Duran A, Collado M, Carrascosa MJ Martin-Caballero J, Flores JM, Diaz-Meco MT, Moscat J and Serrano M: Tumour-suppression activity of the proapoptotic regulator Par4. EMBO Rep 6: 577-583, 2005. 
3. El-Guendy N and Rangnekar VM: Apoptosis by Par-4 in cancer and neurodegenerative diseases. Exp Cell Res 283: 51-66, 2003.

4. Moreno-Bueno G, Fernandez-Marcos PJ, Collado M, et al: Inactivation of the candidate tumor suppressor Par-4 in endometrial cancer. Cancer Res 67: 1927-1934, 2007.

5. Cheema SK, Mishra SK, Rangnekar VM, Tari AM, Kumar R and Lopez-Berestein G: Par-4 transcriptionally regulates Bcl-2 through a WT1-binding site on the bcl-2 promoter. J Biol Chem 278: 19995-20005, 2003

6. Wang G, Reed E and Li QQ: Apoptosis in prostate cancer: progressive and therapeutic implications (Review). Int $\mathrm{J}$ Mol Med 14: 23-34, 2004.

7. Gurumurthy S and Rangnekar VM: Par-4 inducible apoptosis in prostate cancer cells. J Cell Biochem 91: 504-512, 2004

8. Zhao Y, Burikhanov R, Qiu S, Lele SM, Jennings CD Bondada S, Spear B and Rangnekar VM: Cancer resistance in transgenic mice expressing the SAC module of Par-4. Cancer Res 67: 9276-9285, 2007.

9. El-Guendy N, Zhao Y, Gurumurthy S, Burikhanov R and Rangnekar VM: Identification of a unique core domain of par-4 sufficient for selective apoptosis induction in cancer cells. Mol Cell Biol 23: 5516-5525, 2003.

10. Goswami A, Qiu S, Dexheimer TS, Ranganathan P, Burikhanov R, Pommier Y and Rangnekar VM: Par-4 binds to topoisomerase 1 and attenuates its DNA relaxation activity. Cancer Res 68: 6190-6198, 2008.

11. Baker AH: Targeting AAV vectors. Mol Ther 7: 433-434, 2003.

12. Sugita T, Yoshikawa T, Mukai Y, et al: Improved cytosolic translocation and tumor-killing activity of Tat-shepherdin conjugates mediated by co-treatment with Tat-fused endosome-disruptive HA2 peptide. Biochem Biophys Res Commun 363: 1027-1032, 2007.

13. Stachler MD, Chen I, Ting AY and Bartlett JS: Site-specific modification of AAV vector particles with biophysical probes and targeting ligands using biotin ligase. Mol Ther 16: 1467-1473, 2008.

14. Shaner NC, Patterson GH and Davidson MW: Advances in fluorescent protein technology. J Cell Sci 120: 4247-4260, 2007.

15. Jemal A, Murray T, Ward E, Samuels A, Tiwari RC, Ghafoor A, Feuer EJ and Thun MJ: Cancer statistics, 2005. CA Cancer J Clin 55: 10-30, 2005.

16. Ying-Chun S, Chiun $\mathrm{H}$ and Ann-Lii C: Molecular targeted therapy for advanced hepatocellular carcinoma. Targ Oncol 2: 199-210, 2007.
17. Boosen M, Vetterkind S, Koplin A, Illenberger S and Preuss U: Par-4-mediated recruitment of Amida to the actin cytoskeleton leads to the induction of apoptosis. Exp Cell Res 311: 177-191, 2005.

18. Sugita T, Yoshikawa T, Mukai Y, et al: Comparative study on transduction and toxicity of protein transduction domains. Br J Pharmacol 153: 1143-1152, 2008

19. Nori A and Kopecek J: Intracellular targeting of polymer-bound drugs for cancer chemotherapy. Adv Drug Delivery Rev 57: 609-636, 2005.

20. Chauhan A, Tikoo A, Kapur AK and Singh M: The taming of the cell penetrating domain of the HIV Tat: myths and realities. J Control Release 117: 148-162, 2007.

21. Simon MJ, Gao S, Kang WH, Banta S and Morrison B III: TAT-mediated intracellular protein delivery to primary brain cells is dependent on glycosaminoglycan expression. Biotechnol Bioeng 104: 10-19, 2009.

22. Wadia JS, Stan RV and Dowdy SF: Transducible TAT-HA fusogenic peptide enhances escape of TAT-fusion proteins after lipid raft macropinocytosis. Nat Med 10: 310-315, 2004

23. He C, Lao WF, Hu XT, Xu XM, Xu J and Fang BL: Anti-liver cancer activity of TNF-related apoptosis-inducing ligand gene and its bystander effects. World J Gastroenterol 10: 654-659, 2004.

24. Manuel AFVG, Maria GP, Antoine AFV and Dinko V: Generation of a high-capacity hybrid vector: packaging of recombinant adenoassociated virus replicative intermediates in adenovirus capsids overcomes the limited cloning capacity of adenoassociated virus vectors. Virology 288: 236-246, 2001.

25. Teramoto S, Ishii T, Matsuse $\mathrm{T}$ and Fukuchi Y: Recombinant adeno-associated virus vectors efficiently transduce foreign gene into bovine aortic endothelial cells: comparison with adenovirus vectors. Jpn J Pharmacol 84: 206-212, 2000.

26. Yueping L, Shudong Q, Liping S, Qingfeng Y and Guangxiao Y: Secretory expression of $\mathrm{p} 53$ (N15)-Ant following lentivirusmediated gene transfer induces cell death in human cancer cells. Cancer Invest 26: 28-34, 2008.

27. Andrew PH, Stephen JM, Nabil GS and Richard AM: Neurotrophin-4, alone or heterodimerized with brain-derived neurotrophic factor, is sorted to the constitutive secretory pathway. J Biol Chem 278: 48129-48136, 2003. 